DOI: https://doi.org/10.11144/Javeriana.upsy16-4.epef

\title{
Evaluación psicométrica de la escala de felicidad de Lima en una muestra mexicana
}

\section{Psychometric Evaluation of the Happiness Scale of Lima in a} Mexican Sample

\author{
Gabriela Rodríguez-HernándeZ \\ Universidad Autónoma del Estado de México, México \\ ORCID: http://orcid.org/0000-0003-2821-9672 \\ Galileo Domínguez-Zacarías \\ Universidad Autónoma del Estado de México, México \\ María del Consuelo Escoto Ponce de \\ LEÓN \\ Universidad Autónoma del Estado de México, México
}

a Autor de correspondencia. Correo electrónico: grodriguezh@uaemex.mx

Para citar este artículo: Rodríguez-Hernández, G., Domínguez-Zacarías, G., \& Escoto Ponce de León, J. (2017). Evaluación psicométrica de la escala de felicidad de Lima en una muestra mexicana. Universitas Psychologica, 16(4), 1-10. https://doi.org/1 0.11144/Javeriana.upsy16-4.epef

\section{RESUMEN}

México está entre los países más felices del mundo a pesar de sus bajas puntuaciones en prácticamente todos los elementos que proveen bienestar, y requiere de indicadores psicosociales que expliquen la felicidad de los mexicanos. El objetivo de este estudio fue determinar las propiedades psicométricas de la escala de felicidad de Lima (EFL) en una muestra heterogénea mexicana. La EFL considera la felicidad como un comportamiento multidimensional con una importante carga cultural para población latina. Se realizaron análisis factorial exploratorio y análisis factorial confirmatorio mediante el método de ecuaciones estructurales. Los estudios se efectuaron con dos muestras heterogéneas de 600 mexicanos cada una. Los resultados obtenidos llevaron a identificar un modelo bifactorial donde el afecto positivo correlaciona positivamente con la satisfacción con la vida.

\section{Palabras clave}

afecto positivo; satisfacción con la vida; análisis factorial confirmatorio.

\section{ABSTRACT}

Mexico among the happiest countries in the world despite their low scores on virtually all elements that provide well-being, requires psychosocial indicators that explain the happiness of Mexicans. The objective of this study was to determine the psychometric properties of the lime happiness scale (LHS) in a heterogeneous Mexican sample. The LHS considers happiness as a multidimensional behavior with an important cultural burden for the Latino population. Exploratory factorial analysis and confirmatory factor analysis were performed using the structural equations method. The studies were carried out with two heterogeneous samples of 600 Mexicans each. The results obtained led to the identification of a bifactorial model where positive affect correlates positively with satisfaction with life.

Keywords

affection; satisfaction with life; confirmatory factor analysis. 
La felicidad se ha planteado como un estado de ánimo que las personas vigorosas manifiestan como síntoma de buena salud (Seligman, 2011; Seligman, Steen, Park, \& Peterson, 2005). Un estado mental que las personas pueden llegar a controlar cognoscitivamente al percibir y concebir tanto a sí mismos como a su mundo como una experiencia de alegría, de satisfacción o de bienestar positivo (Cuadra \& Florenzano, 2003). La felicidad es el grado en que una persona valora de manera favorable la calidad global de su vida, en este sentido, la felicidad o satisfacción vital es un constructo psicológico individual al valorar subjetivamente la propia vida respecto a cómo se considera que debería ser esta; valoración global y con cierta duración (Veenhoven, 2009).

Por razones conceptuales y empíricas, el estudio de la felicidad se ha incluido en los estudios sobre bienestar subjetivo, es decir, como parte de la evaluación subjetiva que cada persona hace de su propia vida como totalidad o de algunas de sus facetas (Diener, 2000; Diener et al., 2010).

La satisfacción con la vida es uno de los componentes del bienestar subjetivo junto con el balance de afectos positivos y negativos (Diener, 1984; Diener, 2000; Diener, Suh, Lucas, \& Smith, 1999). La satisfacción ha sido definida como un estado mental, como el grado en que una persona evalúa la calidad global de su vida (Atienza, Pons, Balaguer, \& García-Merita, 2000; Veenhoven, 1994). Tarazona (2005), menciona que se trata del componente cognitivo del bienestar subjetivo, y lo define como el sentimiento de bienestar en relación consigo mismo y su propia vida. La satisfacción es relativa, al ser una cuestión puramente cognitiva y no reconocer la experiencia afectiva, es el resultado de la comparación entre cómo es la vida en realidad y las concepciones de cómo es que debería ser (Veenhoven, 1994; Atienza et al., 2000).

La dimensión de los afectos positivos se ha trabajado en menor medida al ser un tema de relativa complejidad, pues involucra condicionantes sociales y culturales (Elster, 2002;
Alcalá, Camacho, Giner, Giner, \& Ibáñez, 2006). Argyle, Martin y Crossland (1989), Diener y Eunkook (2003) y Diener et al., (1999) señalan que los afectos son estados de humor; así como emociones que representan las evaluaciones personales de los sucesos que ocurren en la vida de las personas en contextos sociales y culturales determinados. Fredrickson (1998, 2001), señala que las emociones positivas pueden servir para la consecución de metas de vida que las personas se plantean, así como para la búsqueda del bienestar, además permiten construir y reforzar los recursos con los que cuenta la persona. Incluso hay evidencia empírica que sugiere que dicha espiral ascendente predice con gran efectividad el sentido de bienestar de las personas (Fredrickson, 2000, 2002).

Si bien se ha estudiado la felicidad desde el bienestar subjetivo, su definición enfatiza en su naturaleza subjetiva, y mantiene a los seres humanos individuales como los mejores jueces de su propia felicidad (Diener, 1984); las investigaciones han comenzado a dar cuenta de que la felicidad que se utiliza en el discurso diario tiene un significado difuso y algo diferente a lo propuesto por los estudiosos (Dinner $\&$ Biswas-Diener, 2008). Por lo que en este estudio se recupera la propuesta de Alarcón (2006), quien conceptualiza la felicidad como un estado afectivo de satisfacción plena que experimenta el individuo en posesión de un bien deseado. Esta definición conlleva los siguientes indicadores: a) la felicidad significa sentimientos de satisfacción que experimenta la persona y solo ella en su vida interior, b) el hecho de que sea un estado de la conducta, alude al carácter temporal de esta, c) la felicidad supone la posesión de un bien, es decir, se es feliz en tanto se posee el bien u objeto que hace la felicidad, d) el bien o bienes que la generan son de naturaleza variada (materiales, éticos, estéticos, psicológicos, religiosos, sociales, etc.). Propone estudiar la felicidad como un constructo multidimensional, además de considerar el aspecto cultural latino para la conceptualización y operacionalización de la felicidad.

Por otro lado, México es considerado como uno de los países más felices del mundo, pues en 
diversos estudios ha puntuado muy por arriba del promedio en felicidad (Diener \& Eunkook, 2003; Figueroa, 2013; Fuentes \& Reto, 2011; Instituto Nacional de Estadística y Geografía (INEGI), 2015; Olivares, 2015). Los datos revelan que la felicidad para los mexicanos se relaciona con valores tradicionales como la familia y el amor, mientras los ingresos económicos o el puesto de trabajo son menos importantes. Adicionalmente, en el más reciente Informe Mundial sobre Felicidad (Helliwell, Layard, \& Sachs, 2015) México ocupa la posición 12 de 158 de los países más felices. Además, la Organización para la Cooperación y el Desarrollo Económico (OCDE, 2015) lo ubica como uno de los países más felices, a pesar de su mal desempeño en prácticamente todos los elementos que proveen bienestar, y al ubicarse en los últimos lugares en educación y habilidades; último lugar en seguridad personal; entre los últimos en empleos, ganancias y balance trabajo-vida, pero a la pregunta directa iqué tan feliz es?, el promedio se encuentra en 7.2 en una escala del 0 a 10, lo que lo ubica por encima del promedio de los 34 países que participan en el estudio. Actualmente, la felicidad se considera como indicador de progreso social y un objetivo de política pública de gran cantidad de gobiernos, entre ellos México.

Por lo anterior, cobra importancia ampliar los conocimientos sobre el tema de la felicidad en general, y en particular para los mexicanos, por lo cual el objetivo de este estudio es determinar las propiedades psicométricas de la escala de felicidad de Lima (Alarcón, 2006) en una muestra heterogénea mexicana.

La EFL reporta correlaciones ítem-escala altamente significativas en todos los ítems $(r=0.511 ; p<0.001)$, así como alta consistencia interna $(\alpha=0.92)$. El análisis factorial de componentes principales y rotación varimax puso en evidencia que la felicidad es un comportamiento multidimensional, integrado por cuatro factores: Factor 1. Sentido positivo de la vida, sus ítems indican ausencia de estados depresivos, pesimismo y de vacío existencial. Factor 2. Satisfacción con la vida, los reactivos de este factor transcriben estados subjetivos de satisfacción por la posesión de los bienes deseados. Factor 3. Realización personal, expresa felicidad plena y no estados temporales de estar feliz, así como autosuficiencia; finalmente el Factor 4. Alegría de vivir, refiere lo maravillosa que es la vida, las experiencias positivas y sentirse generalmente bien.

Los estudios sobre el uso y propiedades psicométricas de la EFL son prometedores, ejemplo de ello, es el estudio realizado por Álvarez (2012), quien converge con Alarcón (2006) sobre la estructura multifactorial de la felicidad, y con una teorización igualmente multidimensional de esta. Por su parte, Rodríguez (2010) utilizó la EFL en centros peri-urbanos y urbanos marginales de la ciudad de Lima, en donde reportó una consistencia interna de 0.91. Árraga y Sánchez (2012) validaron la EFL en adultos mayores venezolanos, y reportaron una alta consistencia interna $(0.84)$; sin embargo, a partir de la validez del constructo, modificaron la estructura original de la escala, de la que solo quedaron 14 ítems de los 27 propuestos originalmente, pero manteniendo los cuatro factores propuestos. En México, Toribio, González, Valdez, González y Van Baneveld, (2012), validaron la escala en adolescentes estudiantes de bachillerato entre los 14 y 19 años, pertenecientes a un nivel socioeconómico medio, quienes reportaron una alta consistencia interna (0.91), y confirmaron la estructura de la escala con dimensiones y número de reactivos.

Es importante atender a varias elecciones estadísticas problemáticas, identificadas tanto en la propuesta original como en los estudios posteriores, por ejemplo: a) el autor usó Análisis de Componentes Principales (ACP) para la determinación de las dimensiones, empero, para escalas nuevas. Worthington y Wittaker (2006) recomiendan utilizar análisis factorial confirmatorio (AFC); b) los autores realizaron rotación varimax, la cual es apropiada cuando los factores subyacentes a un grupo de ítems no están correlacionados; c) para determinar el número de factores retenidos, Alarcón (2006) empleó los valores propios mayores a la unidad, lo cual puede sobreestimar/subestimar el número de factores retenidos (Fabrigar, Wegener, MacCallum, \& Strahan, 1999), más aún. Toribio et al. (2012) 
no especifican claramente los criterios utilizados para la retención de factores; y d) se retuvieron los ítems con cargas factoriales mayores a 0.4 , como lo recomiendan los expertos (Worthington $\&$ Wittaker, 2006), sin embargo, no es posible observar los datos sobre los ítems problemáticos, es decir, aquellos con diferencia de cargas menores a 0.15 . Por lo anterior, AFC mediante el modelamiento de ecuaciones estructurales, aportará a la validación de esta escala y a la especificación de los indicadores de cada factor, y con ello, permitirá determinar las propiedades psicométricas de la escala de felicidad de Lima (EFL) en una muestra mexicana.

\section{Método}

\section{Participantes}

Mediante muestreo intencional, un total de 1200 habitantes del Estado de México, de ambos sexos (610 hombres y 590 mujeres), con un rango de edad de entre 18 a 50 años $(M=28.99$; $D E=9.08)$, participaron de manera voluntaria en este estudio. Para los fines de este trabajo, la muestra total se dividió de manera aleatoria en dos submuestras. La muestra 1, sirvió para llevar a cabo el análisis factorial exploratorio (AFE) (Estudio 1), el cual se compuso de 600 participantes (278 mujeres y 322 hombres) con una edad promedio de 29 años $(D E=9.22)$. Con la muestra 2, se realizó el análisis factorial exploratorio (AFE) mediante el modelamiento de ecuaciones estructurales (Estudio 2) la cual se conformó de 600 participantes (323 mujeres y 277 hombres) con una edad promedio de 28 años $(D E=8.94)$.

\section{Instrumentos}

La Escala de Felicidad de Lima (Alarcón, 2006) consta de 27 ítems tipo Likert $(1=$ totalmente en desacuerdo, $5=$ totalmente de acuerdo) con alta consistencia interna $(\alpha=0.92)$. Consta de cuatro factores: sentido positivo de la vida, satisfacción con la vida, realización personal y alegría de vivir, con consistencias internas que van de 0.72 a 0.88 .

\section{Procedimiento}

Después de obtener consentimiento informado, la EFL fue administrada colectivamente en espacios públicos del Estado de México. Los participantes debieron responder datos referentes a edad, sexo y lugar de procedencia.

\section{Análisis estadístico}

Con la muestra 1 , primeramente se realizaron análisis descriptivos así como de correlación para garantizar que no hubiera multicolinealidad. Posteriormente, se realizó un AFE mediante el método de extracción de ejes principales, utilizando para la retención de factores tanto la estrategia de eigenvalue mayor a 1 (ev $>1)$, como el análisis del gráfico de sedimentación. Se utilizó el criterio de rotación oblicua (PROMAX, Kappa =4) (Costello \& Osborne, 2005; Fabrigar et al., 1999; Tabachnick \& Fidell, 2007; Worthington \& Whittaker, 2006). Lo anterior se llevó a cabo con el programa SPSS versión 20.

Posteriormente, con la muestra 2 se realizó AFC de la EFL mediante análisis encuadrado en los modelos de ecuaciones estructurales (Structural, Equation Models [SEM]) con los programas LISREL 8.80 y AMOS 18, con la intención de presentar dos diferentes métodos de estimación de análisis, dado que utilizan diferentes algoritmos de solución, lo que proporcionará una mayor sensibilidad al modelo.

Atendiendo a la naturaleza de las variables, se utilizaron como input las matrices de correlaciones de Pearson. Tomando en cuenta el tamaño de la muestra, el método de estimación empleado fue el método de máxima verosimilitud (Bentler, 1995), y los criterios utilizados para evaluar el ajuste de los modelos fueron, además del chi-cuadrado $\left(\chi^{2}\right)$, el índice de ajuste no normativo (NNFI), el índice de ajuste comparativo (CFI) y la raíz del promedio del error de aproximación (RMSEA). En el caso del 
NNFI y CFI se consideran valores aceptables por encima de 0.9 (Hu \& Bentler, 1998). Para la RMSEA, se consideran aceptables valores inferiores a 0.1 (satisfactorio $\leq$ a 0.08). Para evaluar la diferencia de ajuste, se evaluaron los índices de bondad de ajuste incrementales, y se optó por el modelo más parsimonioso (Chen, 2007).

\section{Resultados}

Estudio 1: Análisis factorial exploratorio (AFE)

Los estadísticos descriptivos obtenidos en la muestra 1 mostraron que existe normalidad univariada de los datos. La validación de la normalidad fue a través del estudio y análisis de la curtosis y asimetría (Curran, West, \& Finch, 1996). Los datos mostraron que todas las variables tienen un valor de asimetría menor que 2 , y los de curtosis son menores que 7 . La fiabilidad para la escala total fue muy alta $(\alpha=$ 0.91).

En la tabla 1, se presentan medias, desviaciones estándar, correlaciones y coeficientes de fiabilidad por factor de la muestra 1 , los cuales se aprecian aceptables $(\alpha>0.7)$ (Nunnally, 1978).

\section{TABLA 1}

Datos descriptivos, correlaciones y de fiabilidad en la muestra 1

\begin{tabular}{|c|c|c|c|c|c|c|c|}
\hline & $M$ & $D E$ & $\begin{array}{l}\text { Sentido } \\
\text { positivo }\end{array}$ & $\begin{array}{l}\text { Satisfacción } \\
\text { con la vida }\end{array}$ & $\begin{array}{c}\text { Realización } \\
\text { personal }\end{array}$ & $\begin{array}{l}\text { Alegría de } \\
\text { vivir }\end{array}$ & Escala total \\
\hline Sentido positivo & 3.83 & 0.76 & $(0.87)$ & & & & \\
\hline Satisfacción con la vida & 3.77 & 0.65 & $0.39^{* *}$ & $(0.83)$ & & & \\
\hline Realización personal & 3.53 & 0.69 & $0.46^{*}$ & $0.69^{\prime \prime}$ & $(0.73)$ & & \\
\hline Alegria de vivir & 3.89 & 0.75 & $0.57^{*}$ & $0.54 "$ & $0.58^{\circ "}$ & (0.79) & \\
\hline scala tot & 3.76 & 0.58 & $0.86^{\circ}$ & $0.74^{\prime \prime}$ & $.79^{* *}$ & .78 & \\
\hline
\end{tabular}

** La correlación es significativa al nivel 0.01 (bilateral). Coeficientes alfa de Cronbach se presentan entre paréntesis. Fuente: elaboración propia.

Respecto del AFE para la EFL, la medida de adecuación muestral de Kaiser-Meyer-Olkin fue excelente $(\mathrm{KMO}=0.9$; test de esfericidad de Bartlet $p<0 \leq 0$ ). Los resultados del AFE mostraron la existencia de cinco factores que explican el 50.12 de la varianza total acumulada, discorde al modelo propuesto por
Alarcón (2006), quien propone cuatro factores. Utilizando 0.40 como criterio de saturación (Tabachnick \& Fidell, 2007), los reactivos se agruparon de la siguiente manera: En el factor 1 se agruparon las variables $3,1,4$, y 9 con saturaciones altas. El factor 2, concentró los reactivos $20,19,22,7$ y 14 . El factor 3, agrupó las variables $21,26,23,2$ y 18 . El factor 4, concentró los ítems 25,24 y 27 , y en el factor 5 se agruparon las variables 16,12 y 13 . El resto de los reactivos $(5,6,17,10,11,8$ y 15$)$ saturaron por encima de 0.4 en más de un factor, lo que indica que no discriminan entre los distintos componentes de la felicidad teorizados (ver tabla 2). Si bien, los resultados del AFE mostraron la existencia de cinco factores que explican el $50.12 \%$ de la varianza total. El análisis del gráfico de sedimentación, se observa que los tres últimos factores no aumentan prácticamente la varianza.

\section{TABLA 2}

Estructura factorial de la Escala de Felicidad

\begin{tabular}{|c|c|c|c|c|c|}
\hline \multirow{2}{*}{ Items } & \multicolumn{5}{|c|}{ Factor } \\
\hline & F1 & $\mathrm{F} 2$ & $\mathrm{~F} 3$ & $\mathrm{~F} 4$ & F5 \\
\hline 03. Las condiciones de mi vida son excelentes & 0.88 & & & & \\
\hline 01. En la mayoria de las cosas mi vida está cerca de mi ideal & 0.78 & & & & \\
\hline 04. Estoy satisfecho con mi vida & 0.63 & & & & \\
\hline 09. Si volviera a nacer, no cambiaría casi nada en mi vida & 0.45 & & & & \\
\hline 20. La felicidad es para algunas personas, no para mi & & 0.87 & & & \\
\hline 19. Me siento un fracasado. & & 0.85 & & & \\
\hline 22. Me siento triste por lo que soy & & 0.5 & & & \\
\hline 07. Pienso que nunca seré feliz & & 0.49 & & & \\
\hline 14. Me siento inútil & & 0.44 & & & \\
\hline 21. Estoy satisfecho con lo que ahora he alcanzado & & & 0.75 & & \\
\hline 26. Todavia no he encontrado sentido a mi existencia & & & 0.73 & & \\
\hline 23. Para mi la vida es una cadena de sufrimientos & & & 0.7 & & \\
\hline 02. Siento que mi vida está vacia & & & 0.68 & & \\
\hline 18. Tengo problemas tan hondos que me quita $h$ tranquilidad & & & 0.61 & & \\
\hline 25. Mi vida transcurre plácidamente & & & & 0.76 & \\
\hline 24. Me considero una persona realizada & & & & 0.73 & \\
\hline 27. Creo que no me falla nada & & & & 0.46 & \\
\hline 16. He experimentado la alegria de vivir & & & & & 0.71 \\
\hline 12. Es maravilloso vivir & & & & & 0.65 \\
\hline 13. Por lo general me siento bien & & & & & 0.53 \\
\hline Varianza & 30.56 & 10.55 & 3.80 & 2.85 & 2.36 \\
\hline
\end{tabular}

Estudio 2: Análisis factorial confirmatorio (AFC)

Los estadísticos descriptivos (media, desviación típica, asimetría y curtosis) obtenidos para cada una de las variables, arrojaron valores estadísticos que apuntan a la normalidad univariada de los datos. Los resultados mostraron que todas las variables tienen un valor de asimetría menor que 2, y los valores de curtosis menores que 7. La fiabilidad para la escala total fue muy 
alta $(\alpha=0.91)$. En la tabla 3, se presentan medias, desviaciones estándar, correlaciones y coeficientes de fiabilidad por factor de la muestra 2 , los cuales se aprecian aceptables $(\alpha>0.7)$ (Nunnally, 1978).

\section{TABLA 3}

Datos descriptivos, correlaciones y de fiabilidad en la muestra 2

\begin{tabular}{|c|c|c|c|c|c|c|c|}
\hline & M & $D E$ & $\begin{array}{l}\text { Sentido } \\
\text { positivo }\end{array}$ & $\begin{array}{c}\text { Satisfacción } \\
\text { con la vida }\end{array}$ & $\begin{array}{c}\text { Realización } \\
\text { personal }\end{array}$ & $\begin{array}{c}\text { Alegría de } \\
\text { vivir }\end{array}$ & $\begin{array}{c}\text { Escala } \\
\text { total }\end{array}$ \\
\hline Sentido positivo & 3.83 & 0.75 & $(0.87)$ & & & & \\
\hline Satisfacción con la vida & 3.77 & 0.67 & $0.38^{* *}$ & $(0.82)$ & & & \\
\hline Realización personal & 3.51 & 0.68 & $0.39^{* *}$ & $0.63^{* *}$ & $(0.74)$ & & \\
\hline Alegria de vivir & 3.86 & 0.74 & $0.59^{* *}$ & $0.56^{*}$ & $0.58^{* *}$ & $(0.76)$ & \\
\hline Escala total & 3.75 & 0.57 & $0.85^{*}$ & $0.74^{*}$ & $0.75^{* *}$ & $0.8^{* *}$ & $(0.91)$ \\
\hline
\end{tabular}

La tabla 4 muestra los valores de los índices de bondad de ajuste para la EFL, y evidencia que si bien, en la literatura correspondiente no se aprecia acuerdo sobre cuáles han de ser los valores de los índices considerados y medidas para un buen ajuste, la mayor parte de estas medidas están comprendidas entre el rango de cero (mal ajuste) a uno (ajuste perfecto). Primeramente se puso a prueba el modelo teórico, es decir, el de cuatro factores, el cual no presentó un ajuste satisfactorio. Tomando como base los resultados obtenidos en el AFE, también se pusieron a prueba los modelos de cinco y dos factores. El primero de ellos exhibió ajustes no satisfactorios, no así el modelo de dos factores. Por tanto, los resultados del AFC confirman los resultados obtenidos en el AFE, toda vez que el modelo de dos factores presentó un buen ajuste en los dos métodos: [0.05 $\left.<\mathrm{X}^{2} \leq 1\right]$, $[0 \leq$ RMSEA $\leq 0.05]$, $[0.95 \leq \mathrm{NNFI} \leq 1]$ y $[0.97 \leq$ $\mathrm{CFI} \leq 1]$.

\section{TABLA 4}

Índices de Bondad LISRELL y AMOS

\begin{tabular}{lcccccc}
\hline Índice & \multicolumn{2}{c}{ Modelo 4 factores } & \multicolumn{2}{c}{ Modelo 5 Factores } & \multicolumn{2}{c}{ Modelo 2 Factores } \\
\hline Procedimiento & LISREL & AMOS & LISREL & AMOS & LISREL & AMOS \\
\hline $\mathrm{X}^{2}$ & 903.70 & 1120.76 & 473.54 & 473.54 & 45.98 & 45.98 \\
$\mathrm{gl}$ & 309 & 318 & 160 & 160 & 26 & 26 \\
$\mathrm{X}^{2} / \mathrm{gl}$ & 2.92 & 3.52 & 2.96 & 2.96 & 1.77 & 1.77 \\
$\mathrm{NNFI}$ & 0.91 & 0.71 & 0.92 & 0.81 & 0.96 & 0.94 \\
$\mathrm{CFI}$ & 0.94 & 0.77 & 0.94 & 0.87 & 0.98 & 0.97 \\
$\mathrm{RMSEA}$ & 0.08 & 0.09 & 0.07 & 0.08 & 0.04 & 0.05 \\
$\mathrm{AGFI}$ & 0.76 & 0.72 & 0.83 & 0.83 & 0.95 & 0.94 \\
SRMR & 0.06 & 0.09 & 0.07 & 0.07 & 0.05 & 0.05 \\
\hline
\end{tabular}

Fuente: elaboración propia.
La figura 1 representa el modelo de dos factores que presentó el mejor ajuste. En el factor 1, las saturaciones factoriales oscilaron entre 0.5 y 0.73 , y los ítems hacen referencia a la satisfacción con la vida. En el factor 2, las saturaciones factoriales fluctuaron entre 0.56 y 0.84 , y los ítems dan cuenta del sentido positivo de la vida. Todas las saturaciones factoriales fueron significativas $(p<0.01)$. La matriz de correlación reveló una relación significativa y positiva entre los dos factores latentes $(P h i=0.42 ; p<0.01)$. Además, ambos factores mostraron una fiabilidad moderada $(\alpha=0.73$ y $\alpha=0.81)$.

\section{Figura 1}

Modelo estructural de dos factores de la felicidad

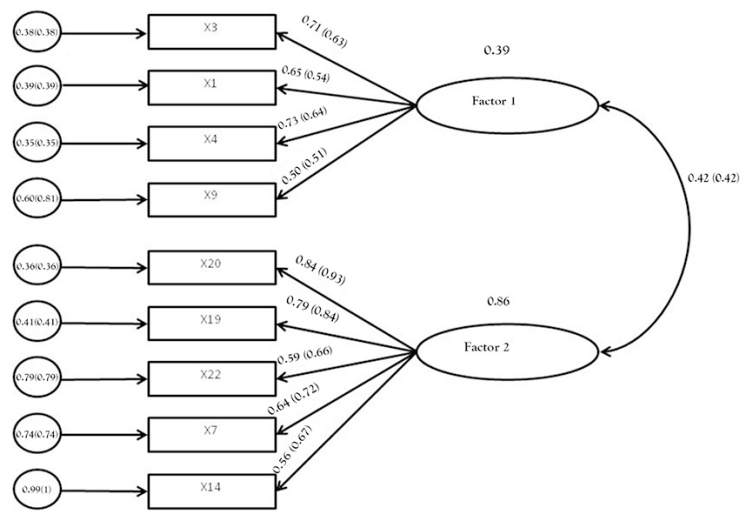

Nota: Los valores dentro del paréntesis corresponden al procedimiento LISRELL, y fuera de éstos los obtenidos en AMOS. Los óvalos denotan las variables exógenas y los rectángulos representan las variables endógenas. Los valores que aparecen junto a las flechas que van desde las variables exógenas hacia las endógenas indican la carga factorial de las variables indicadoras en el factor común. Los valores de los círculos representan el error de medida. Fuente: elaboración propia.

En el factor 2, se aprecian las cargas factoriales más altas, entre las que se encuentran que la felicidad no es solo para algunas personas, sino también para uno mismo $(b=0.93)$, así como no sentirse fracasado $(b=0.84)$, ni triste $(b=0.66)$, por el contrario, pensar que se puede ser feliz $(b=$ $0.72)$, y no sentirse inútil $(b=0.67)$, constituyen la forma en que se ve o percibe la vida para ser feliz.

El factor 1 muestra que pensar que las condiciones de la vida son buenas $(b=0.63)$, 
así como pensar que la mayoría de las cosas de la vida se acercan al ideal personal $(b=0.54)$, lleva a estar satisfecho con la vida $(b=0.64)$ y a no querer cambiar nada de esta $(b=0.51)$. A partir de lo anterior, es que se puede decir que una cantidad importante de la varianza de los indicadores ha sido capturada por los dos constructos, lo que revela evidencia de fiabilidad y validez de las variables latentes.

Estos resultados junto con los obtenidos primeramente en el AFE, llevan a considerar que la felicidad para la muestra de mexicanos se puede explicar principalmente a través de constructos como el sentido positivo y la satisfacción con la vida.

\section{Discusión}

El objetivo de este estudio fue determinar las propiedades psicométricas de la escala de felicidad de Lima (EFL) en una muestra heterogénea mexicana, para lo cual se realizó un AFE (estudio1) y un AFC (estudio 2). Los resultados obtenidos en ambos estudios no confirmaron la estructura de cuatro factores del modelo teórico.

Los resultados sugieren que la diferencia de estructura factorial podría deberse a que el instrumento fue diseñado originalmente con universitarios; y aun cuando en México se validó la escala con adolescentes, y se confirmó la estructura original de la misma (Toribio, et al., 2012). También es cierto que se tomaron elecciones estadísticas problemáticas, mismas que se precisaron con antelación en este estudio. No obstante, coincidimos con Árraga y Sánchez (2012), quienes a partir del análisis psicométrico que hacen a la EFL con adultos mayores venezolanos, modificaron la estructura original de la escala.

Cada uno de los dos factores identificados mediante SEM verifican empíricamente la covariación entre las respuestas a los reactivos que representan afecto positivo y satisfacción con la vida, constructos que teóricamente se han identificado como necesarios para entender la felicidad (Diener, 1984; Diener, 2000; Diener et al., 1999; Fredrickson 1998, 2001). La estructura bifactorial se aprecia suficientemente robusta, pues ambos factores presentan coeficientes alfa que indican una consistencia interna adecuada. El factor con mayor carga es el que representa al afecto positivo. Este factor considera la felicidad como algo asequible a todos, donde no caben los sentimientos de fracaso, de tristeza o de ineptitud. Estas emociones positivas amplían los recursos intelectuales, físicos y sociales de los individuos, y amplían las reservas a las que se puede recurrir cuando se presentan amenazas u oportunidades; asimismo, incrementan los patrones para actuar en ciertas situaciones mediante la optimización de los propios recursos personales en el nivel físico, psicológico y social (Fredrickson, 2001). Por cuanto hace a la satisfacción con la vida, su contribución más alta se ubica en pensar que se está satisfecho con la vida. Por último, la correlación entre los dos factores es positiva y moderada, en consonancia con datos previos (Diener, 1984; Diener et al., 1999; Diener, 2000).

Los datos obtenidos nos permiten decir que la felicidad se percibe no solo como experiencia, sino sobre todo como una actitud, es decir, como una evaluación en la que intervienen elementos afectivos, cognitivos y comportamentales, cuyo origen se ubica en predisposiciones aprendidas socialmente al tener una función adaptativa en un contexto cultural determinado, apreciación en la que se distinguen normas culturales, metas y valores que influyen en la manera en que este grupo de mexicanos seleccionan, interpretan, procesan y usan la información para la construcción del concepto felicidad (Rodríguez-Hernández, Domínguez-Zacarias, \& Lugo, 2016; Rodríguez-Hernández, Juárez \& Cruz, 2016).

A la vista de los resultados, se pueden señalar varias cosas: en primer lugar, se ha conseguido estimar el modelo sin problemas. En segundo lugar, la revisión de los parámetros revela estimaciones razonables. En tercer lugar, los valores de $\mathrm{R}^{2}$ oscilan de 0.25 a 0.7 , lo que sugiere que las variables observadas son indicadores pertinentes de las variables latentes, que describen el constructo felicidad. 
Finalmente, como lo sugieren Juárez (2014) y Escoto et al. (2017), las futuras investigaciones sobre la EFL deben determinar si los hallazgos aquí encontrados son atribuibles a la escala, pues otras mediciones han demostrado su capacidad significativa para medir la felicidad. Los investigadores podrían administrar la EFL a diferentes grupos etarios, y debe ser evaluada su estabilidad a lo largo de períodos más largos. Además, deberán examinar las actuales propuestas teóricas (por ejemplo, la felicidad eudaimónica y hedónica).

\section{Referencias}

Alarcón, R. (2006). Desarrollo de una Escala Factorial para medir Felicidad. Revista Interamericana de Psicología/Interamerican Journal of Psychology, 40(1), 99-106.

Alcalá, V., Camacho, M., Giner, D., Giner, J., \& Ibáñez, E. (2006). Afectos y género. Psicothema, 18(1), 143-148.

Álvarez, L.Y. (2012). Escala de Creencias acerca de la Felicidad en Población Adulta de la ciudad de Bucaramanga (Colombia). Investigación y Desarrollo, 20(2), 302-333.

Argyle, M., Martin, M., \& Crossland, J. (1989). Happiness as a function of personality and social encounters. En J. P. Forgas \& J. M. Innes (Eds.), Recent advances in social psychological: An international perspective (189-203). North-Holland: Elsevier.

Árraga, M. V., \& Sánchez, M. (2012). Validez y confiabilidad de la escalada de Felicidad de Lima en adultos mayores venezolanos. Universitas Psychologica, 11(2), 381-393.

Atienza, F.L., Pons, D., Balaguer, I., \& GarcíaMerita. (2000). Propiedades Psicométricas de la Escala de Satisfacción con la Vida en Adolescentes. Psicothema, 12(2), 314-319.

Bentler, P. M. (1995). EQS Structural equations program manual. Encino, CA: Multivariate Software.

Chen, F. F. (2007). Sensitivity of Goodness of Fit Indexes to Lack of Measurement Invariance. Structural Equation Modeling, 14(3), 464-504.
Costello, A. B., \& Osborne, J. W. (2005). Best practices in exploratory factor analysis: four recommendations for getting the most from your analysis. Practical Assessment, Research, Eु Evaluation, 10, 1-9.

Cuadra, H., \& Florenzano, R. (2003). El bienestar subjetivo: hacia una psicología positiva. Revista de Psicología de la Universidad de Chile, 12(1), 83-96.

Curran, P. J., West, S.G., \& Finch, G.F. (1996). The robustness of test statistics to nonnormality and specification error in confirmatory factor analysis. Psychological Methods, 1, 16-29.

Diener, E. (1984). Subjective well-being. Psychological Bulletin, 95, 542-575.

Diener, E. (2000). Subjective wellbeing: The Science of happiness and proposal for a national index. American Psychologist, 55, 34-43. https:// doi.org/10.1037/0003-066X.55.1.34.

Dinner, E., \& Biswas-Diener, R. (2008). Happiness. Unlocking the mysteries of psychological wealth. USA: Blackwell, Publishing.

Diener, E., \& Eunkook, M.S. (2003). National differences in subjective well-being. En D. Kahneman, E. Diener y N. Schwartz (Eds.): Well-being. The foundations of hedonic psychology (434-450). New York: Russell Sage Foundation.

Diener, E., Helliwell, F.H., \& Kahneman, D. (2010). International Differences in WellBeing. Oxford: University Press, Inc.

Diener, E., Suh, E. M., Lucas, R. E., \& Smith, H. E. (1999). Subjective well-being: Three decades of progress. Psychological Bulletin, 125(2), 276-302. https:// doi.org/10.1037//0033-2909.125.2.276

Elster, J. (2002). Alquimias de la mente. La racionalidad y las emociones. Barcelona: Paidós.

Escoto, C., Bosques-Brugada, L., Camacho, E.J., Alvarez-Rayón, G., Franco, P.K., \& Rodríguez, H.G. (2017). Psychometric evaluation of the muscle appearance satisfaction scale in a Mexican male simple. 
Eating and Weight Disorders, 1-9. https:// doi.org/10.1007/s40519-017-0366-0

Fabrigar, L. R., Wegener, D. T., MacCallum, R. C., \& Strahan, E. J. (1999). Evaluating the use of exploratory factor analysis in psychological research. Psychological Methods, 4(3), 272-299. https:// doi.org/10.1037/1082-989X.4.3.272.

Figueroa, H. (2013). Querétaro, Monterrey y D.F, zonas más felices de México. Excelsior. Recuperado de http://www.excelsior.com. $\mathrm{mx} /$ nacional/2013/02/20/885054.

Fredrickson, B.L. (1998). What good are positive emotions? Review of General Psychology, 2(3), 300-319.

Fredrickson, B.L. (2000) Cultivating positive emotions to optimize health and well-being. Prevention y Treatment, 3. Disponible en línea: http://journals.apa.org/prevention/vo lume3/pre0030001a.html

Fredrickson, B.L. (2001). The role of positive emotions in positive psychology. The broaden-and-build theory of positive emotions. American Psychologist, 56, 218-226.

Fredrickson, B.L. (2002). Positive emotions. En C. R. Snyder y S. J. Lopez (Eds.): Handbook of positive psychology. New York: Oxford University Press.

Fuentes, N.C., \& Reto, F. (2011). Las condiciones de Vida y la felicidad. En García, J.J., \& Sales, F.J. Bienestar y Calidad de Vida en México (pp. 69-112). México: Centro de Estudios Sociales y de Opinión Pública.

Helliwell, J., Layard., R., \& Sachs, J. (2015), Reporte Mundial de Felicidad. Recuperado de http://worldhappiness.report/wp-conten t/uploads/sites/2/2015/04/WHR15.pdf

Hu, L., \& Bentler, P. (1998). Fit Indices in Covariance Structure Modeling: Sensitivity to Underparameterized Model Misspecification. Psychological Methods, 3(4), 424-453.

Instituto Nacional de Estadística y Geografía [INEGI] (2015). Bienestar Subjetivo. Contenido de la BIARE. Recuperado de http://www.inegi.org.mx/inegi/contenid os/investigacion/experimentales/bienestar/ contenido.aspx.

Juárez, C.S. (2014). Propiedades psicométricas del cuestionario Honey-Alonso de estilos de aprendizaje(CHAEA) en una muestra mexicana. Journal of Learning Styles, 7(13), 137-154.

Nunnally, J. C. (1978). Psychometric theory (2nd ed.). New York, NY: McGraw-Hill.

Olivares, E. (2015). Mexicanos tienen alto nivel de satisfacción y felicidad con la vida. La jornada. Recuperado de http://www.jornad a.unam.mx/2015/02/06/sociedad/036n1soc

Organización para la Cooperación y Desarrollo Económico. [OCDE] (2015). Better Life Index. Recuperado de http://www.oecdbett erlifeindex.org/es/

Rodríguez, C. (2010). Variables psicológicas asociadas con la felicidad en centros periurbanos y urbanos marginales de Lima. UCV-Scientia, 2(1), 61-68.

Rodríguez-Hernández, G., Domínguez-Zacarías, G., \& Lugo, C. (2016). Bayesian Posterior Predictive Probability Happiness. Applied Mathematics, 7, 753-764. https:// doi.org/10.4236/am.2016.78068.

Rodríguez-Hernández, G., Juárez, L.C., \& Cruz, C.P. (2016). Análisis Estructural de la Felicidad. La Psicología Social en México, XVI, 533-537.

Seligman, M., Steen, T., Park, N., \& Peterson, C. (2005).

Positive Psychology progress: Empirical validation of interventions. American Psychologist, 60(5), 410-421. https:// doi.org/10.1037/0003-066X.60.5.410172.

Seligman, M. (2011). Flourish. A visionary new understanding of happiness and well-being. New York: Free Press.

Tabachnick, B. G., \& Fidell, L. S. (2007). Using multivariate statistics. Boston: Pearson/Allyn $\&$ Bacon.

Tarazona, D. (2005). Autoestima, Satisfacción con la vida y condiciones de habilidad en adolescentes estudiantes de quinto año de media. Un estudio Factorial según Pobreza y Sexo. Revista IIPSI, 8, 57-65. 
Toribio, L., González, N. I., Valdez, J. L., González, S., \& Van Baneveld, H.O. (2012). Validación de la Escala de Felicidad de Alarcón para adolescentes mexicanos. Psicología Iberoamericana, 20(1), 71-79.

Veenhoven, R. (2009). Medidas de la Felicidad Nacional Bruta. Intervención Psicosocial, 18(3), 279-299.

Veenhoven, R., (1994). El estudio de la satisfacción con la vida. Intervención psicosocial, 3, 87-116.

Worthington, R. L., \& Wittaker, T. A. (2006). Scale Development Research: a content analysis and recommendations for Best practices. The Counseling Psychologist, 34(6), 806-838. https:// doi.org/10.1177/001100000628812

\section{Notas}

* Artículo de investigación. 